Ref.TH.3124-CERN

\title{
WEAK INTERACTIONS, SYMMETRIES
}

\author{
J. Bernabeu \\ CERN - Geneva \\ and \\ Departament de Fisica Teorica, \\ Universitat de Valencia, Spain
}

ABSTRACT

The standard ideas about the breaking of symmetries, through the mechanism of generation mixing, are discussed. New results and proposals looking for discrete space-time symmetry violation as well as flavour-number violation are reported. The use of weak-interaction processes as a tool for both the study of the structure of the currents and for nuclear-structure problems is emphasized.

Invited paper presented at the IX International Conference on High Energy Physics and Nuclear Structure Versailles, 6 - 10 Juily 1981

Ref.TH. $3124-C E R N$

14 July 1981 
WEAK INTERACTIONS, SYMMETRIES

\title{
J. BERNABEU
}

\author{
CERN, Geneva, Switzerland \\ Departament de Física Teörica, Universitat de Valēncia, Spain
}

Abstract: The standard ideas about the breaking of symmetries, through the mechanism of generation mixing, are discussed. New results and proposals looking for discrete space-time symmetry violation as well as flavour-number violation are reported. The use of weak-interaction processes as a tool for both the study of the structure of the currents and for nuclear-structure problems is emphasized.

\section{Introduction}

A first look at the abstracts submitted to this session of the Conference shows a great diversity of topics and the abundant research work covered by this active field. I will try to present, as far as it is possible, some unifying views on the subject.

The title "Weak Interactions, symmetries" indicates a connection between possible symmetries of nature and the studies of processes mediated by weak interactions. The history of physics teaches us the disrespect shown by the weak interactions in nature towards our original prejudices about rigorous symmetries. After the experimental evidence of parity violation twenty-five years ago, Lee and Yang and, independently, Landau suggested a restoration of the lost symmetry emphasizing the invariance under CP. However, the violation of CP invariance observed in 1964 for the system of neutrai kaons invalidated the general principle that the world of particles in front of a mirror behaves identically to the world of antiparticles behind the mirror. Owing to the CPT theorem, one automatically associates CP violation with time-reversal violation. In fact, T violation has been explicitly shown experimentally for the neutrai kaon system, whereas CPT remains a good symmetry.

What about flavour numbers? The deep-seated reason to account for the existence of flavour numbers for hadrons and leptons is still not completely understood. The internal charges of hadrons, such as strangeness, charm, etc., are known to be non-conserved by charged-current weak interactions. In the lepton world, nature seems to be organized in doublets each containing a charged lepton and a neutrino so that a rigorous separate conservation law operates. Is there any reason for these separate lepton numbers? Are all neutrinos massless? A big fraction of papers submitted to this session touch on these problems of violation of symmetries, and I propose to discuss some of them as follows: a) space-time symmetries, parity and time reversal; b) flavour numbers, neutrino mass and muon-number violation.

To provide a convenient framework for our discussion, I shall describe below those ingredients of the standard electroweak theory which are relevant for these problems of violation of symmetries.

Within the field of weak-interaction processes, several recent studies have been directed towards disentangling the structure of the hadronic weak currents: CVC, PCAC, second class, etc. Muon capture in nuclei is also used, in other cases, as a probe for nuclear-structure problems which include nucleonic and mesonic degrees of freedom. So this lecture will contain another section treating the charged-current weak processes, with two subsections: hadronic currents and nuclear structure. 


\section{Standard ideas}

The present dogma establishes that the only exact symmetries of nature are those implemented by local gauge invariance. This implies the existence of gauge bosons, transforming as the adjoint representation of the group of gauge transformations. Electroweak interactions are described ${ }^{1)}$ by the group $S U(2) \times U(1)$, so there are two charged and two neutral massless gauge boson mediators. Conventional weak interactions are, however, observed to be short ranged, so the physical mediators have to be massive objects*). This is accomplished through the mechanism of spontaneous breaking of the gauge symmetry. The ground state of the system is not invariant under the symmetry of the interaction. Things arrange themselves so that the photon $\gamma$ remains massless, whereas the $W^{ \pm}$and the $Z^{0}$ acquire a mass. Therefore, within the same framework, there are electromagnetic, charged-current weak, and neutral-current weak interactions contributing to a given process. In the last few years, the data on neutral-current interactions have shown agreement with this scheme. The neutral-current couplings extracted from neutrino scattering and parity-violating electron scattering are as given by the standard theory; in particular, the global strength of the neutral-current interaction tests the mechanism of spontaneous symmetry breaking.

The building blocks of matter are the fermion fields of leptons and quarks, described by their flavour numbers. They transform as a given representation of the group. Putting the left-handed components in doublets, the flavours appear in several generations

$$
\left(\begin{array}{c:c}
v_{e} & u \\
e^{-} & d
\end{array}\right), \quad\left(\begin{array}{c:c}
\nu_{\mu} & c \\
\mu^{-} & s
\end{array}\right), \quad\left(\begin{array}{l:l}
\nu_{\tau} & t \\
\tau- & b
\end{array}\right)
$$

with a fundamental lepton-quark symmetry being shown. The concept of generation is associated with theory repeating itself for each doublet of flavours. As already sajd, within a generation the left-handed components transform as doublets under SU(2), whereas the right-handed components are singlets. Therefore, the fermion currents contain simuitaneously vector and axial pieces, and PARITY VIOLATION appears from the start. Charged currents are $V-A$, and weak neutral currents are parity violating too. This has been demonstrated experimentally in the beautiful $\mathrm{e}^{-\mathrm{d}}$ experiment at SLAC, and indications have been presented in atomic physics.

Electroweak interactions depend on flavour, in such a way that $y$ and $Z^{0}$ currents are diagonal, whereas $W^{ \pm}$currents connect vertically the two members of a generation. At this level, one should talk about the generation-number conservation. For leptons, this corresponds to the separate conservation of lepton number. For hadrons, it would correspond to the absence of Cabibbo-like rotations. However, the horizontal structure of the theory is not understood; there is no good reason to stop the number of generations. With several generations, we have a serious problem. How do we know that the weak SU(2) eigenstates coincide with the flavour states of definite mass? The most general choice corresponds to

$$
\begin{array}{ll}
u^{\prime}{ }_{j} L=A_{j k}^{(u, L)} u_{k} L, & u^{\prime}{ }_{j}^{R}=A_{j k}^{(u, R)} u_{k} R \\
d^{\prime}{ }_{j} L=A_{j k}^{(d, L)} d_{k}{ }^{L}, & d^{\prime}{ }_{j}^{R}=A_{j k}^{(d, R)} d_{k} R,
\end{array}
$$

where $j, k=1,2, \ldots, n$ if there are $n$ generations and $u, d$ refer to the two components of each generation.

The change of basis corresponds to a unitary matrix connecting the flavours $u_{j}\left(d_{j}\right)$ to the weak states $u^{\prime}{ }_{j}\left(d^{\prime}{ }_{j}\right)$. It is a consequence of this unitarity property that, in the standard theory, the electromagnetic and neutral-current weak interactions are independent of the choice of $u^{\prime}, d^{\prime}$. They connect $u \rightarrow u, d \rightarrow d$ only.

*) Other possibilities can be envisaged, such as in hypercolour theories. 
It is said that natural flavour conservation operates for neutral currents. Vertices such as $s \rightarrow d, \mu \rightarrow e$ are not present in the theory, although transitions of this kind could be induced through higher orders.

What about charged-current interactions? This sector behaves completely differently. The vertical connection produces a charged current

$$
\sum_{j} \bar{d}_{j}^{\prime} \gamma^{\mu}\left(1+\gamma_{5}\right) u_{j}^{\prime}=\sum_{j, k} d_{j} \gamma^{\mu}\left(1+\gamma_{5}\right)\left[A(d, L)+A_{A}(u, L)\right]_{j k} u_{k}
$$

and the matrix $U \equiv A^{(d, L)+} A(u, L)$ is not the identity 1 . Looking at Eq. (2), one concludes that there could be generation-number violation by charged currents, with vertices

$$
s \rightarrow u, \quad \mu^{-}+v_{1}, \ldots .
$$

What is not forbidden is allowed! For hadrons, we have been acquainted with this fact: it corresponds to the Cabibbo rotation, which gives rise to the $\Delta s=1$ currents. Why then insist on the separate conservation of lepton numbers?

Let me concentrate, first, on the hadron sector putting $U \neq \mathbf{1}$. A simple reasoning tells us that, for a $n \times n$ unitary matrix $U$, there are physically $n(n-1) / 2$ moduli and $(n-1)(n-2) / 2$ phases as independent parameters. If $n=2$, only one real parameter remains. With two generations, the original Cabibbo rotation is the most general scenario for this mechanism of generation-number violation. For $n=3$ generations, however, there are three moduli and one phase which are relevant parameters, and which are conventionally chosen à la Kobayashi-Maskawa ${ }^{2}$. It is interesting to speculate that the relative phase present here is responsible for the origin of $T$ violation. To incorporate $C P$ violation was the motivation of these authors to extend the theory to three generations. In this framework, the manifestation of $T$ violation needs that the three generations come into play for a given process. This explains why this phenomenon, contrary to parity violation, is so elusive. In particular, for nuclear physics studies, T violation could only appear as a higher order electroweak correction to processes in which only ordinary $(u, d)$ quarks are relevant to leading order.

It is economical that, for hadrons, the same mechanism that produces flavour violation in charged-current weak interactions can explain CP violation. Is there a parallel situation for leptons? We have emphasized before that the origin of the matrix $U \neq \mathbb{1}$ is to be found in the fact that the eigenstates of weak interactions do not coincide with the flavour (definite mass) eigenstates. In the standard theory, in which masses are created through couplings to the scalar Higgs particles, the mass matrix is non-diagonal on the bas is of weak eigenstates ${ }^{*}$. The corresponding diagonalization is what leads to the definite flavour states. To see what can happen for leptons, let me take the case of two generations. The effects of the matrix $U \neq 1$, in charged currents, are contained in the Cabibbo rotation, which can be chosen to act on the neutrino states. Thus, the neutrinos active in weak interactions correspond to the states

$$
\begin{aligned}
& v_{e}=\cos \theta v_{1}-\sin \theta v_{2} \\
& \nu_{\mu}=\sin \theta v_{1}+\cos \theta v_{2} .
\end{aligned}
$$

But, if both neutrinos $v_{1}$ and $\nu_{2}$ were massless (or degenerate), the angle $\theta$ would be unobservable: there is no way to identify what $v_{1}$ and $v_{2}$ are. In that case, the only objects to be seen experimentally would be $v_{e}$ and $v_{\mu}$, the ones present in weak interactions. If, however, $v_{1}$ and $v_{2}$ were massive (non-degenerate) one would

*) Apart from the non-diagonal mass-matrix mechanism for flavour-number violation, this can occur owing to flavour-changing gauge bosons and/or Higgs particle couplings. 
have the same situation as for the hadron sector. One realizes why in this mechanism the neutrino mass problem is connected with the question of muon-number violation. This concludes our discussion on the present views about the violation of symmetries. These ideas provide a convenient unified description, al though there is no deep understanding of the generation problem.

\section{Space-time symmetries}

\subsection{PARITY}

The implications of the $V$ - A structure of weak charged currents and the predicted parity violation by neutral currents in the standard theory are discussed in several contributions to this Conference. This covers leptonic and semileptonic, as well as non-leptonic processes.

There have been measurements ${ }^{j}$ ) at SIN of the $\mathrm{e}^{+}$polarization from $\mu^{+}$decay, using fast-counter techniques. The Iongitudinal component of the polarization was found to be $P_{L}=1.010 \pm 0.064$, consistent with the $V$ - A prediction. The authors have considered the measurement of a possible transverse component. For semileptonic processes, the reaction ${ }^{12} \mathrm{C}\left(\mu^{-}, \nu\right)^{12} \mathrm{~B}$ has been studied in a complete experiment by the ETH-Louvain group ${ }^{4}$ ). They have measured the average and longitudinal recoil polarizations. Once the nuclear form factors are determined, the result for $P_{L}$ determines the helicity of the neutrino. They get for the muon neutrino helicity $h_{V}=-1.08 \pm 0.11$, as expected from the $V-A$ theory.

A theoretical investigation of parity violation in electron inelastic scattering from deuterium, $e^{-}+d \rightarrow e^{-}+p+n$, has been reported. The authors ${ }^{5}$ have compared the contributions coming from the semileptonic neutral-current weak interaction ( $Z^{0}$ exchange) and from nuclear parity-violating effects through $\gamma$ exchange. They conclude that, for medium-energy electrons,. the asymetry is dominated by weak neutral currents. For low-energy electrons, however, the second contribution becomes comparable to the first. It would be interesting to see whether both ingredients could be extracted experimentally. Thus, apart from the important information on neutral-current couplings, an indirect check of the Lobashov experiment ${ }^{6}$ ) could be made. The theoretical approach to the parity violation in $n p \rightarrow d y$ is still a matter of controversy: factorization, meson approach to the weak NN force, quark approach to the core region, etc.

Experiments aimed at determining the strength of the weak hadronic axial isoscalar current in deuterium atoms have been proposed. Again, there are effects from a parity-violating component in the nucleus which can simulate a genuine axial isoscalar neutral current. Desplanques ${ }^{7}$ ) argues that the isovector $N N$ weak force present in the spin contribution of the $\mathrm{NNY}$ interaction might be the most important correction. The theoretical expectations have to be clarified, however ${ }^{a}$.

The lack of theoretical understanding of the parity-violating nucleon-nucleon interaction is now apparent from the fact that the measured ${ }^{9}$ ) asymmetry in pp scattering, for a proton $1 \mathrm{ab}$. momentum of $6 \mathrm{GeV} / \mathrm{c}$, is much 1 arger (one order of magnitude!) than a] conventional calculations ${ }^{10}$ ), making use of the "best values" proposed by $\left.D D H^{11}\right)$ for the effective weak couplings. One has to realize that this situation cannot come as a surprise. Why do non-leptonic $\Delta S=0$ weak interactions have to be in better shape than $\Delta S \neq 0$ or $\Delta C \neq 0$ transitions? There is at present considerable activity on trying to understand the possible mechanisms for these non-leptonic decays. In nuclear physics studies, it would be interesting to connect the effective operators to other processes in prder to test the basic ingredients. Recently, a nice relation has been found ${ }^{12}$ ) between the mesonic correction to the axial charge operator $\vec{\sigma} \cdot \vec{p}$ and the pion-exchange component of the parity non-conserving nucleon-nucleon potential.

A new field of activity corresponds to parity non-conserving neutron-spin rotation at threshold $\left(E_{n} \leqslant 10^{-2} \mathrm{eV}\right)$

$$
\mathrm{n}+\mathrm{N} \rightarrow \mathrm{n}+\mathrm{N} \text {, }
$$

which has been suggested as a tool to provide direct information on the strength of the neutron-nuclear weak interaction. The presence of a parity non-conserving 
amplitude fPNC for forward scattering induces two effects:

a) Im $f_{P N C}$ gives different total cross-sections for the two helicity states of the neutron.

b) Re fPNC causes the two helicity states to accumulate different phases upon passage through the medium. A neutron with spin initially transverse $|+\rangle+|-\rangle$ leaves the medium in spin state $e^{i \phi[}\left[|+\rangle+e^{i \Delta|-\rangle]}\right.$, where $\Delta$ is the signal of parity violation.

First experimental results ${ }^{13}$ ) have been obtained for tin isotopes, with the following values for the spin rotation in $10^{-6} \mathrm{rad} / \mathrm{cm}$ :

$$
\begin{aligned}
& { }^{124} \mathrm{Sn}:+0.48 \pm 1.49, \\
& { }^{117} \mathrm{Sn}:+36.7 \pm 2.7, \\
& \text { natural } \mathrm{Sn}:+4.95 \pm 0.93
\end{aligned}
$$

with the positive sign corresponding to a right-handed rotation about the momentum. There is no understanding of the source of the enhanced effects in ${ }^{117} \mathrm{Sn}$. First estimates were based on the existence of $p$-wave resonances near threshold, which enhance the strong scattering volume and induce effects in fPNC through the DWBA. The case of ${ }^{124} \mathrm{Sn}$ was suggested theoretically, and the results are rather puzzling. Apart from the "direct" neutron-nuclear weak interaction, there is a source of parity violation with the strong interaction of the neutron with parity impurities in the target nucleus. An interesting approach would be the comparison with the neutron-capture $\gamma$-ray asymmetry, also large in ${ }^{117} \mathrm{Sn}: A_{Y}=(4.4 \pm 0.6) \times 10^{-4}$.

\subsection{TIME REVERSAL}

The search for a non-vanishing electric dipole moment of the neutron has produced very stringent tests for the origin of time-reversal invariance violation. Latest experimental results ${ }^{14}$ give an upper limit $d_{n}<6 \times 10^{-25} \mathrm{e} \cdot \mathrm{cm}$.

The renewed interest and activity in time-reversal studies in nuclear physics comes from the observation ${ }^{15}$ ) of a failure of the relation between the measured proton polarization in

$$
{ }^{7} \mathrm{Li}\left({ }^{3} \mathrm{He}, \overrightarrow{\mathrm{p}}\right){ }^{9} \mathrm{Be} \text { and }{ }^{9} \mathrm{Be}\left({ }^{3} \mathrm{He}, \overrightarrow{\mathrm{p}}\right){ }^{1{ }^{1} \mathrm{~B}}
$$

and the asymetry in the angular distribution for the inverse reactions.

Furthermore, a discrepancy between the measured cross-sections for the two inverse reactions

$$
p+d \underset{+}{p} y+{ }^{3} \mathrm{He}
$$

persists ${ }^{16}$ ) at the level of factors of two. It is imperative to clarify the issue and test the detailed balance theorem. New experimental results in the energy region of the $\Delta(1232)$ resonance have been presented at this Conference, and new measurements are being planned. A look at the present situation seems to indicate that the problem is an experimental one, since there are conflicting results among different groups within the same reaction. It would be interesting to try to figure out the origin of the present experimental discrepancies.

It goes without saying that if time-reversal violation were found at the level of magnitude in these experiments which is currently being discussed, its origin could not be the one described above from the mixing of generations in the Kobayashi-Maskawa matrix.

\section{Flavour numbers}

In the leptonic sector, these studies correspond to testing the separate conservation law of lepton numbers. As discussed in Section 2, this problem is related in the standard theory to the existence of non-degenerate massive neutrinos, 
so we discuss some results (and tests which have been proposed) for both searches at the same time.

There is an improved upper limitit) for muonium $\left(\mu^{+} \mathrm{e}^{-}\right)$conversion to antimuonium $\left(\mu^{-} e^{+}\right.$), which at the $95 \%$ c. 7 . implies $G<42 G_{F}$, where $G$ is the coupling constant for a possible muon-number violating interaction between muons and electrons and $G_{F}$ is the weak Fermi coupling constant. With standard ideas in mind, this conversion does not seem to be a sensitive test of lepton-number violation. The process has been studied to test for the multiplicative lepton-number scheme. Asano et al. 18) have presented at this Conference the results of a search for a heavy neutrino emitted in

$$
k^{+} \rightarrow \mu^{+} v
$$

decay, measuring at KEK (Japan) the muon-range spectrum in stopped $\mathrm{K}^{+}$decays. In this experiment, the possible interference among different flavour (definite mass) states is destroyed, so that the analys is of the experiment is made as follows. The mixing ratio $\left|U_{\mu x}\right|^{2}$ between the $\mu$ generation and any other $x$ generation is given as function of the neutrino mass for that generation. I reproduce the bound found by these authors. They are sensitive to a range of neutrino masses around $200 \mathrm{MeV}$.

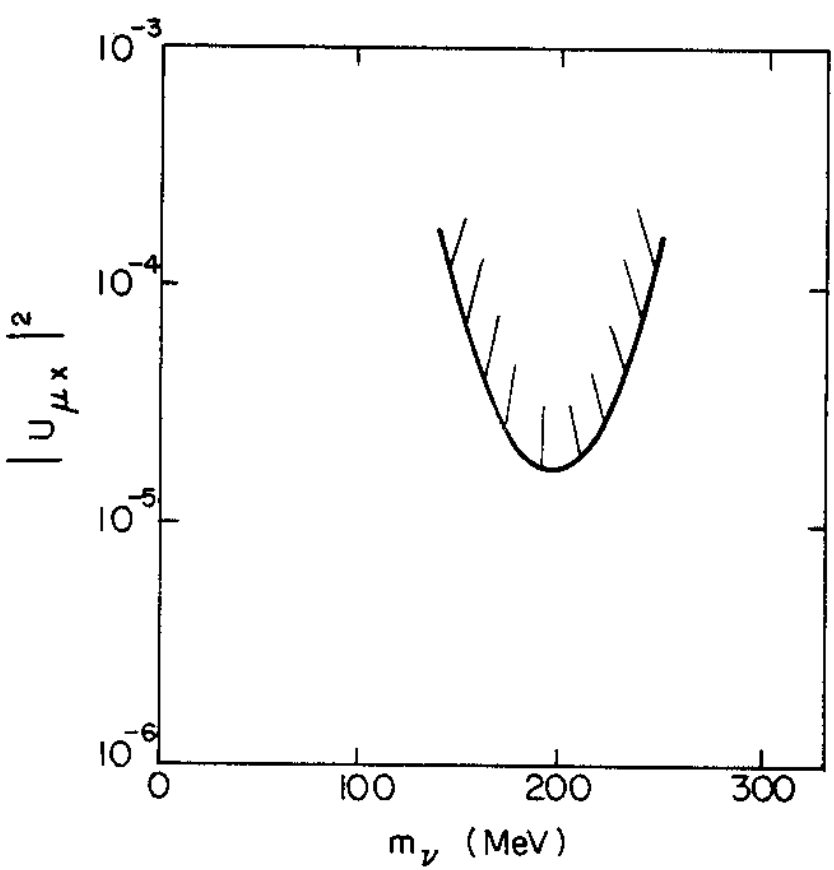

Similar arguments are being used to make this analysis from the reaction $\pi^{+} \rightarrow \mu^{+} \nu$ in an experiment ${ }^{i g}$ ) at SIN.

Present ]imits on [or evidence ${ }^{20}$ ) for !] "electron" neutrino masses come from the $\beta$ decay of ${ }^{3} \mathrm{H}$, and new experiments on radiative electron capture have been proposed $^{21}$ ) and undertaken. There is a proposal submitted ${ }^{22}$ ) to this conference, in which partial nuclear transitions in the radiative muon-capture process are suggested to improve the limit on the "muon" neutrino mass. The shape of the high-energy part of the gamma spectra is fully controlled by phase space, but that part sensitive to the neutrino mass amounts to only B.R. $\sim 10^{-9}, 10^{-10}$, where B.R. is the branching ratio with respect to the total disappearance rate of the muon. Another possibility ${ }^{23}$ ) comes from the pion radiative decay $\pi^{+}+\mu^{+} \nu \gamma$, measuring the photon end-point energy and/or the rate integrated over the extreme upper right corner region of the photon energy. One should remember that, in at1 these cases, one tests an incoherent sum of processes allowed by phase space with definite neutrino masses. 
The effects discussed in the above paragraph, as well as the phenomenon of neutrino oscillations, do not imply that the neutrino has to be considered necessarily as a massive Dirac fermion. Because neutrinos have no electric charge, a left-handed neutrino $\nu_{L}$ can be the eigenstate of charge conjugation and there can be a Majorana mass term $m_{V} V_{L} V_{L}$, without the presence of a right-handed neutrino. A Majorana mass term has a change of lepton number $\Delta L=2$, so that neutrinoless double-beta decay becomes allowed. From the existing experimental bounds on neutrinoless decays of ${ }^{82} \mathrm{Se}$ and ${ }^{76} \mathrm{Ge}$, Haxton et al.$^{24}$ ) have produced a Majorana mass limit $\langle\mathrm{m}\rangle_{\nu} \lesssim 15 \mathrm{eV}$.

\section{Weak processes}

\section{5.] CVC, PCAC, 2nd CLASS, etc.}

In this Section some ideas of how to test the structure of the weak hadronic current are discussed. A stringent test of the Conserved Vector Current (CVC) hypothesis, in its strong version, can be obtained from the branching ratio of the pion $\beta$ decay

$$
\pi^{+} \rightarrow \pi^{0} \mathrm{e}^{+} \mathrm{ve}_{\mathrm{e}}
$$

which is being measured ${ }^{25}$ ) at Los Alamos with $3 \%$ statistics. This decay presents some advantages over the superallowed Fermi $B$ transitions $0^{+}-0^{+}$in nuclei, because a better control of radiative corrections is expected.

One of the quantitatively open questions for the weak nucleonic current is the magnitude of the induced pseudoscalar form factor $g p\left(q^{2}\right)$, which is predicted by the Partially Conserved Axial Current (PCAC) to be $g_{p}(\mu)(P C A C)=-8.4$ at a value of $q^{2}$ associated with the reaction

$$
\mu^{-}+p \rightarrow \nu+n
$$

Bardin et a $1 .^{26}$ ) have recently reported a measurement at Saclay of the muon-capture rate in liquid hydrogen, obtained by comparing the $\mu^{-}$and $\mu^{+}$lifetimes when stopped in matter. Recently ${ }^{27}$ ), the same authors have observed the transition from the ORTHO to the PARA state of the pup molecule, through the non-exponential time distribution for the capture neutrons. The observed rate has therefore to be corrected for the admixture of $\sim 10 \%$ of PARA state. A reanalysis of the result, assuming $V-A$ currents, CVC, no second class, and $\mu-\mathrm{e}$ universality, leads to

$$
g_{p}^{(\mu)}=-8.2 \pm 2.4
$$

in good agreement with the theoretical expectation. Preliminary results on the muon-capture rate by deuterons are expected soon, using a similar technique.

Nuclear PCAC has been verified in the ETH-Louvain experiment $\left.{ }^{4}\right)$, measuring simultaneously the average and longitudinal polarizations of recoil in the process ${ }_{12} \mathrm{C}\left(\mu^{-}, \nu\right){ }^{12} \mathrm{~B}$. Their ratio is almost insensitive to corrections for captures to $12 \mathrm{~B}^{*}$ states. The result for the nuclear pseudoscalar-axial ratio of form factors yields

$$
F_{P}^{(\mu)} / F_{A}^{(\mu)}=-1.03 \pm 0.14
$$

to be compared with the PCAC prediction of -0.99 .

An anomalous G-parity axial-vector term in the weak hadronic current, for which there is some counter evidence in nucleonic transitions, can be probed ${ }^{28}$ ) in pion radiative decay $\pi \rightarrow$ evr. It adds to the first-class axial term either constructively or destructively, depending on the charge state of the decaying pion. If, in either pion charge state, the circular polarization of the photon were measured the vector and axial terms could be separately determined. This would allow a test 
of strong CVC, by comparing the vector contribution with the well-known process $\pi^{0} \rightarrow r$

The Omicron Spectrometer group at the CERN Synchro-cyclotron has observed ${ }^{29}$ ) the rare decay

$$
\pi^{0} \rightarrow \mathrm{e}^{+} \mathrm{e}^{-}
$$

which is expected to proceed via $2 \gamma$ exchange. There is, in addition, a neutralcurrent interaction ( $Z^{\circ}$ exchange), which tests the axial isovector neutral current.
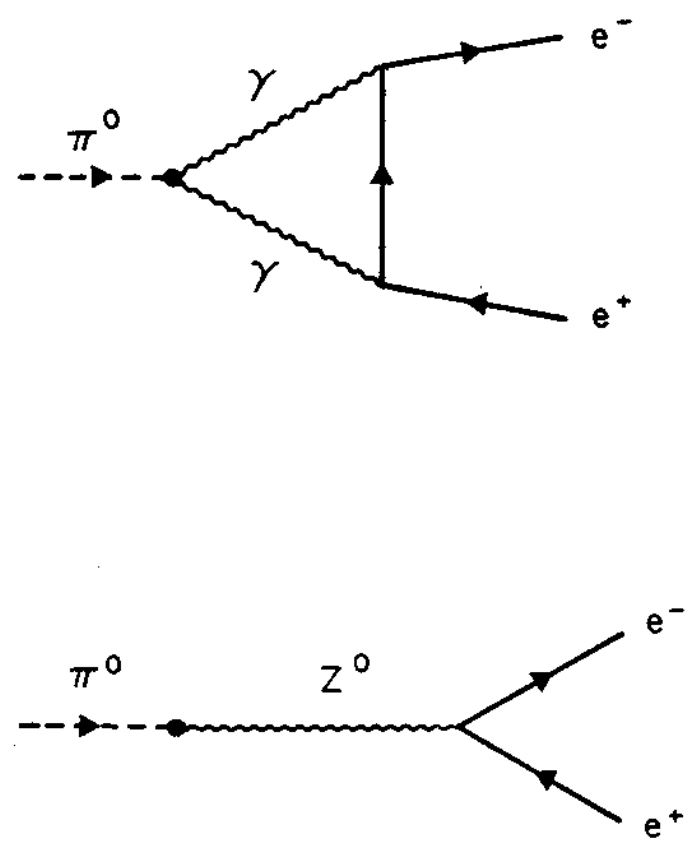

Unfortunately, its effects are prorional to the electron mass. First experimental results for the branching:, indicate that it is appreciably larger than the unitarity lower bound of $5 \times$, coming from the imaginary part of $2 \gamma$ exchanges.

\subsection{NUCLEAR STRUCTURE}

The use of weak-interaction processes with a nucleus as a tool for nuclearstructure studies is the subject of many investigations. One tests the microscopic descriptions of the nucleonic and mesonic degrees of freedom of the nuclear system. Among the contributions presented at this Conference, there are studies on total and partial muon-capture rates, neutron emission after nuclear muon capture, etc.

The experimental material accumulated on total muon-capture rates is rather abundant. New measurements, with better precision using the lifetime technique, have been made ${ }^{30}$ ) for ${ }^{6} \mathrm{Li}$ and ${ }^{7} \mathrm{Li}$ and are being planned for ${ }^{4} \mathrm{He}$ and ${ }^{3} \mathrm{He}$. Two distinct approaches can be followed:

a) The closure approximation, in $i$ ts improved form ${ }^{31}$ ), determines the total strength from the explicit inputs of the ground-state description and the nuclear Hamiltonian. A systematic analys is has been carried out ${ }^{32}$ ) for nuclei in the $1 p$ shell, showing the sensitivity of the energy-weighted sum rule to the effective interaction. The set of parameters of the nuclear Hamiltonian is restricted to reproducing Cohen-Kurath's two-body matrix elements. Results are in very good agreement with experiment at the middle of the she $17\left({ }^{10} \mathrm{~B},{ }^{12} \mathrm{C}\right)$, and somewhat higher for ${ }^{26} 0$. This is understood from RPA-type contributions to the non-energy weighted sum. b) The strength distribution, or response function, is calculated in a detailed model going through each of the final states. Nguyen Van Giai et al. ${ }^{3}$ ) have presented a multipole distribution for the vector strength of muon capture in 
$4^{\circ} \mathrm{Ca}$ and ${ }^{16} 0$, calculated in a continuum-RPA model. The Skyrme force SIII is used. Assuming approximate equality between vector, axial, and pseudoscalar contributions to total capture rates, they find a good agreement with measured values.

Measurements of $\mu^{-}$partial capture rates in ${ }^{10} \mathrm{~B},{ }^{12} \mathrm{C},{ }^{14} \mathrm{~N}$, and ${ }^{16} 0$ have been performed ${ }^{34}$ ) by Giffon et a1., using the short pulses of the Saclay Linac. The rates to bound excited states are deduced from the intensities of the observed deexcitation $\gamma$-rays. The data have been interpreted ${ }^{35}$ ) in terms of the impulse approximation plus mesonic exchange-currents.

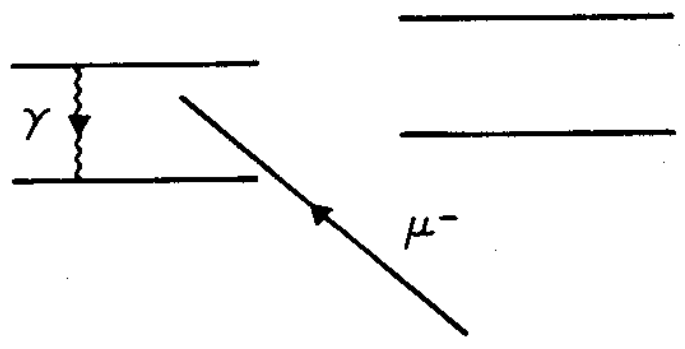

One of the puzzling phenomena in nuclear muon capture is the emission of very energetic neutrons, not predicted by naive impulse approximation studies. Bertl et al. ${ }^{36}$ ) are performing, at SIN, measurements of

i) the neutron-energy spectra

ii) the energy dependence of the neutron asymmetry

iii) neutron-neutron angular correlations after capture by ${ }^{40} \mathrm{Ca}$ and ${ }^{12} \mathrm{C}$. These measurements, improving the experimental situation, will provide interesting information on the mechanism responsible for large energy transfer in muon capture.

\section{Conclusions}

The diversity of topics touched upon in this Session on Weak Interactions, symmetries, should not obscure some unifying ideas.

The detailed knowledge of the symmetries broken by weak interactions is the way to clarify the "family" problem. It is a preliminary step to trying to answer questions such as why the muon exists. Muon-number violation, neutrino masses, time-reversal violation, etc., are ali interconnected problems within the standard approach. Parity violation is present within the electroweak theory from the start, but our understanding of the $\Delta S=0$ non-leptonic weak interactions relevant for nuclear physics is stili far from being satisfactory. Charged-current, neutralcurrent and strong interactions come into play.

Semileptonic weak processes in nuclei are a powerful tool to determine the structure of the hadronic current, with its induced terms. In a microscopic description of the nucleus, the axial-transition strength provides a way to understanding the nucleonic and mesonic nuclear degrees of freedom.

I close with the optimistic view that (at least some of) the open questions that remain after this Conference will have an answer on the next occasion, in two years from now.

\section{Acknowledgements}

I would like to thank the Programme Committee of the Conference, in particular Philippe Catillion and Pierre Radvanyi, for the kind invitation extended to me. Clarifying discussions with M. Ericson, T. Ericson, E. Fiorini, C. Jarlskog and $S$. Noguera are acknowledged. I thank the CERN Theoretical Studies Division for hospitality. 
References

1) S.L. Glashow, Nucl. Phys. 22, 579 (1961).

S. Weinberg, Phys. Rev. Lett. 19, 1264 (i967).

A. Salam, in Proc. 8th Nobel symposium (ed. N. Svartholm) (Almqvist and Wiksel1, Stockholm, 1968).

2) M. Kobayashi and T. Maskawa, Prog. Theor. Phys. $\underline{49}, 652$ (1973).

3) F. Corriveau et al., Abstract M22, 9th Int. Conf. on High-Energy Physics and Nuclear Structure, Versailles, 1981.

4) L. Ph. Roesch et a1., Abstract M26, 9th Int. Conf. on High-Energy Physics and Nuclear Structure, Versailles, 1981.

5) E.M. Henley et al., Abstract M3, 9th Int. Conf. on High-Energy Physics and Nuclear Structure, Versailles, 1981.

6) V.M. Lobashov et aT., Nucl. Phys. AT97, 241 (1972).

7) B. Desplanques, Abstract M8, 9th Int. Conf. on High-Energy Physics and Nuclear Structure, Versailles, 1981.

8) E.M. Henley and W.Y.P. Hwang, Phys. Rev. 23, 1001 (1981).

9) N. Lockyer et a]., 20th Int. Conf. on High-Energy Physics, Madison, 1980.

10) See, for example, A. Barroso and D. Tadić, Abstract M2, 9 th Int. Conf. on HighEnergy Physics and Nuclear Structure, Versailles, 1981.

11) B. Despianques et a1., Ann. Phys. (NY) 124, 449 (1980).

12) W.C. Haxton, Phys. Rev. Lett. $\underline{46}, 698$ (1981).

13) M. Forte et al., Phys. Rev. Lett. $\underline{45}, 2088$ (1981). 14) I.S. Altarev et al., Leningrad Nuclear Physics Institute preprint No. 636
(1981).

15) R.J. Slobodrian, C. Rioux, R. Roy, H.E. Conzett, P. von Rossen and F. Hinterberger, to be published.

16) See Abstracts M7, M1 1 and M12, 9th Int. Conf. on High-Energy Physics and Nuclear Structure, Versailles, 1981.

17) G.M. Marshall et a1., Abstract M23, 9th Int. Conf. on High-Energy Physics and Nuclear Structure, Versailles, 1981.

18) Y. Asano et al., Abstract M18, 9th Int. Conf. on High-Energy Physics and Nuclear Structure, Versailles, 1981.

19) R. Abela et al., Abstract M40, 9th Int. Conf. on High-Energy Physics and Nuclear Structure, Versailles, 1981.

20) V.A. Lyubimov et al., Phys. Lett. $\underline{948}, 266$ (1980).

21) A. De Rujula, preprint CERN-TH.3045 (1981). 22) R.A. Eramzhyan et a1., Abstract M37, 9 th Int. Conf. on High-Energy Physics and
Nuclear Structure, Versailles, 1981 . 
23) J. Missimer et al., Abstract M41, 9th Int. Conf. on High-Energy Physics and Nuclear Structure, Versailles, 1981.

24) W.C. Haxton et al., Abstract M43, 9th Int. Conf. on High-Energy Physics and Nuclear Structure, Versailles, 1981.

25) F.H. Cverna et aT., Abstract MT9, 9th Int. Conf. on High-Energy Physics and Nuclear Structure, Versailles, 1981.

26) G. Bardin et al., Nucl. Phys. A352, 365 (1981).

27) G. Bardin et al., Abstract M35, 9th Int. Conf. on High-Energy Physics and Nuclear Structure, Versailles, 1981.

28) F. Scheck and R. Tegen, Abstract M20, 9th Int. Conf. on High-Energy Physics and Nuclear Structure, Versailles, 1981.

29) W. van Doesburg et al., Abstract M21, 9th Int. Conf. on High-Energy Physics and Nuclear Structure, Versailles, 1981.

30) G. Bardin et al., Saclay preprint, submitted to Phys. Rev. Lett.

31) J. Bernabêu, Nucl. Phys. A201, 41 (1973).

32) J. Navarro et a1., Abstract M36, 9th Int. Conf. on High-Energy Physics and Nuclear Structure, Versailles, 1981.

33) Nguyen Van Giai et al., Abstract M32, 9th Int. Conf. on High-Energy Physics and Nuclear Structure, Versailles, 1981.

34) M. Giffon et a1., CEA N-2204, to appear in Phys. Rev. C.

35) P. Guichon and C. Samour, Abstract J12, 9th Int. Conf. on High-Energy Physics and Nuclear Structure, Versailles, 1981.

36) W. Bertl et al., Abstract M33, 9th Int. Conf. on High-Energy Physics and Nuclear Structure, Versailles, 1981. 
